\title{
On the number of crepant valuations of canonical singularities
}

\author{
Mirel Caibăr
}

\begin{abstract}
In this note we calculate the number of crepant valuations of an isolated canonical singularity $0 \in X:(f=0) \subset \mathbb{C}^{n}$, which is assumed to be nondegenerate with respect to its Newton polyhedron, in terms of weightings and the Newton polyhedron of $f$.
\end{abstract}

\section{Introduction}

Let $0 \in X:(f=0) \subset \mathbb{C}^{n}$ be (a germ of) an isolated canonical hypersurface singularity. Given a morphism $\varphi: Y \rightarrow X$ from a partial resolution $Y$ with at most terminal singularities, we can write

$$
K_{Y}=\varphi^{*} K_{X}+\sum a_{i} E_{i}
$$

where $E_{i}$ are the $\varphi$-exceptional prime divisors and $a_{i} \geq 0$. The discrepancy $a_{i}$ depends only on the valuation of the divisor $E_{i}$. If $a_{i}=0$ the divisor $E_{i}$ is called crepant. A crepant valuation $v$ has centre a divisor $E_{i}$ on any partial resolution $Y$ of $X$ with at most terminal singularities. The number of crepant valuations $c(X):=\#\left\{i: a_{i}=0\right\}$, measuring how far is $X$ from being terminal, is finite and independent of $Y$.

Under the assumption that the hypersurface $X$ is nondegenerate with respect to its Newton polyhedron, we develop in this note the calculations in toric geometry enabling us to detect the birationally defined divisors $E_{i}$ in terms of weightings and the Newton polyhedron of $f$; in particular, we derive a formula for their number, $c(X)$.

The arguments work for a $n$-dimensional hypersurface $X$, but we are mainly interested in the 3 -fold case. If $\varphi: Y \rightarrow X$ is a minimal model of $X$, then $c(X)$ is equal to the fourth Betti number of $Y$. The problem of

\footnotetext{
${ }^{0}$ Mathematics Subject Classification 2000: 14B05, 14J17
} 
determining the cohomology of $Y$ was studied in [1], and will be the subject of a forthcoming paper.

In Section 2 we recall standard terminology and notation from toric geometry.

For a weighting $\alpha$, denote by $\alpha(\mathbf{1})$ the sum of the weights, and by $\alpha(f)$ the degree of the $\alpha$-tangent cone of $X$. In Section 3 we use a discrepancy calculation from [4], and note that every crepant valuation is represented by an exceptional divisor on an $\alpha$-blowup $\varphi_{\alpha}: X(\alpha) \rightarrow X$, where $\alpha$ is a weighting satisfying the equation $\alpha(\mathbf{1})=\alpha(f)+1$; we call the weightings satisfying this equation crepant, and denote the set of crepant weightings by $W(f)$. This gives a relation between crepant valuations and exceptional prime divisors on weighted blowups with crepant weightings.

In order to make this correspondence 1-to-1, we define in Section 4 the set $E(f)$ consisting of the exceptional prime divisors $E$ on $\alpha$-blowups of $X$ with $\alpha$ crepant satisfying $v_{E}\left(x_{i}\right)=\alpha_{i}$, where $v_{E}$ is the valuation of $E$. Using some auxiliary results from the previous section, we obtain a characterisation of the set $E(f)$ (Theorem 4.3). This gives a list of representatives of the crepant valuations, which are certain hypersurfaces in weighted projective spaces.

As a consequence, we obtain in Section 5 a formula for $c(X)=\# E(f)$ in terms of the set $W(f)$ of crepant weightings and the Newton diagram $\Gamma(f)$ of $f$. Namely

$$
c(X)=\sum_{\substack{\alpha \in W(f) \\ \operatorname{dim} \Gamma_{\alpha}=1}} \operatorname{length} \Gamma_{\alpha}+\#\left\{\alpha \in W(f): \operatorname{dim} \Gamma_{\alpha} \geq 2\right\},
$$

where $\Gamma_{\alpha}$ denotes the face of $\Gamma(f)$ corresponding to $\alpha$, and length $\Gamma_{\alpha}=$ $\#\left(\mathbb{Z}^{n} \cap \Gamma_{\alpha}\right)-1$.

\section{Acknowledgements}

This paper is part of my University of Warwick Ph.D. thesis. It is a great pleasure to thank my research advisor, Miles Reid, for his constant support and guidance. This work is a reflection of his ideas, explanations and criticism during many hours of mathematical discussions.

The final version of this paper was written during a postdoctoral position at University of North Carolina at Chapel Hill. I thank both institutions for hospitality, and Alessio Corti, David Mond and Jonathan Wahl for useful conversations. 


\section{Preliminaries}

Recall standard toric terminology. Let $M$ be the free Abelian group $\mathbb{Z}^{n}$, $N=\operatorname{Hom}_{\mathbb{Z}}(M, \mathbb{Z})$ its dual, $M_{\mathbb{R}}=M \otimes_{\mathbb{Z}} \mathbb{R}$ and $N_{\mathbb{R}}=N \otimes_{\mathbb{Z}} \mathbb{R}$ the vector spaces obtained by extending scalars to $\mathbb{R}$. We identify $m \in M$ with the monomial $x^{m}=\prod_{i=1}^{n} x_{i}^{m_{i}}$. A weighting $\alpha=\left(\alpha_{1}, \ldots, \alpha_{n}\right) \in N$ is primitive if the $\alpha_{i}$ have no common factor.

Let $e_{1}, \ldots, e_{n}$ denote the standard basis of $N_{\mathbb{R}}, \sigma=\sum_{i=1}^{n} \mathbb{R}_{\geq 0} e_{i} \subset$ $N_{\mathbb{R}}$ the positive quadrant, and $\sigma^{\vee}=\sum_{i=1}^{n} \mathbb{R}_{\geq 0} e_{i}^{*}$ the dual quadrant. It is convenient to write $m \in f$ if $m$ appears in the polynomial $f=\sum_{m \in M} a_{m} x^{m}$ with $a_{m} \neq 0$. For each $\alpha \in \sigma \cap N$, define then $\alpha(m)=\sum_{i=1}^{n} \alpha_{i} m_{i}$ and

$$
\alpha(f)=\min _{m \in f} \alpha(m) .
$$

Let $0 \in X:(f=0) \subset \mathbb{C}^{n}$ be an isolated hypersurface singularity. The Newton polyhedron of $f, \Gamma_{+}(f)$, is the convex hull in $M_{\mathbb{R}}$ of the set $\bigcup_{m \in f}\left(m+\sigma^{\vee}\right)$; the union of all its compact facets, $\Gamma(f)$, is called the Newton diagram of $f$. For any face $\Gamma \prec \Gamma(f)$ denote $f_{\Gamma}=\sum_{m \in \Gamma \cap M} a_{m} x^{m}$. The polynomial $f$ is nondegenerate with respect to its Newton polyhedron if, for each face $\Gamma$ of $\Gamma(f)$, the hypersurface defined by $f_{\Gamma}=0$ is nonsingular on $\left(\mathbb{C}^{*}\right)^{n}$. The defining property of a nondegenerate hypersurface $X$ is that it can be desingularised by a suitable toric resolution of the ambient space $\mathbb{C}^{n}$.

Consider the filtration of ideals $\mathcal{I}_{\bullet}$ on $A=\mathbb{C}\left[x_{1}, \ldots, x_{n}\right]$ given by

$$
\mathcal{I}_{k}=\{g \in A: \alpha(g) \geq k\} .
$$

Let $\mathcal{A}:=\bigoplus_{k \geq 0} \mathcal{I}_{k}$ be the blowup algebra of $\mathcal{I}_{\bullet}$ in $A$, and $\varphi_{\alpha}: \mathbb{C}^{n}(\alpha):=$ $\operatorname{Proj} \mathcal{A} \rightarrow \mathbb{C}^{n}$ the weighted blowup of $\mathbb{C}^{n}$ with weights $\alpha_{1}, \ldots, \alpha_{n}$. The corresponding graded ring

$$
g r_{\mathcal{I}} A:=\bigoplus_{k \geq 0} \mathcal{I}_{k} / \mathcal{I}_{k+1}
$$

defines the exceptional set by $E_{\alpha}:=\operatorname{Proj} g r_{\mathcal{I}} . A \cong \mathbb{P}(\alpha)$. The standard open subsets of $\operatorname{Proj} \mathcal{A}$ are

$$
U_{j}=\operatorname{Spec} \mathbb{C}\left[\sigma_{j}(\alpha)^{\vee} \cap M\right],
$$

where $\sigma_{j}(\alpha)=\sum_{i \neq j} \mathbb{R}_{\geq 0} e_{i}+\mathbb{R}_{\geq 0} \alpha$.

If there exists $j$ such that $\alpha_{j}=1$, then a minimal set of generators for $\sigma_{j}(\alpha)^{\vee} \cap M$ is $\left\{e_{j}^{*}, e_{i}^{*}-\alpha_{i} e_{j}^{*}\right.$ for $\left.1 \leq i \leq n\right\}$, and thus

$$
U_{j}=\operatorname{Spec} \mathbb{C}\left[x_{j}, y_{1}, \ldots, \hat{y}_{j}, \ldots, y_{n}\right] \cong \mathbb{C}^{n},
$$


where $y_{i}=x_{i} / x_{j}^{\alpha_{j}}$ for all $1 \leq j \leq n$ with $j \neq i$. In general, $U_{j}=\mathbb{C}^{n} / \mu_{\alpha_{j}}$, where the group $\mu_{\alpha_{j}}$ of primitive $\alpha_{j}$ th roots of unity acts on $\mathbb{C}^{n}$ by

$$
x_{j} \mapsto \varepsilon x_{j} \quad \text { and } \quad y_{i} \mapsto \varepsilon^{-\alpha_{i}} y_{i}
$$

If $\alpha \in \sigma \cap N$ is a primitive weighting, we can avoid working on the cyclic cover by choosing a different open set of $\operatorname{Proj} \mathcal{A}$. Let $m_{0} \in M$ be a monomial with $\alpha\left(m_{0}\right)=1$, as in $[4,(4.8)]$, and $u=x^{m_{0}}$. Consider then

$$
U_{\alpha}=\operatorname{Spec} \mathbb{C}\left[u, y_{1}, \ldots, y_{n}\right],
$$

where $y_{i}=x_{i} / u^{\alpha_{i}}$ for $1 \leq i \leq n$. On this open set, the $\alpha$-blowup is given by

$$
x_{i}=u^{\alpha_{i}} y_{i} \text { for } 1 \leq i \leq n .
$$

\section{Crepant weightings}

Let $0 \in X:(f=0) \subset \mathbb{C}^{n}$ be an isolated canonical nondegenerate singularity. For each primitive weighting $\alpha \in \sigma \cap N$, denote by $\varphi_{\alpha}: X(\alpha) \rightarrow X$ the $\alpha$ blowup of $X$.

It is shown in [4, Section 4.8] that, for any primitive weighting $\alpha \in \sigma \cap N$, there is a relation

$$
K_{\mathbb{C}^{n}(\alpha)}+X(\alpha)=\varphi_{\alpha}^{*}\left(K_{\mathbb{C}^{n}}+X\right)+(\alpha(\mathbf{1})-\alpha(f)-1) E_{\alpha},
$$

where $\mathbf{1}=(1, \ldots, 1) \in M$. In particular, the divisor $E_{\alpha}$ is crepant with respect to the pair $\left(\mathbb{C}^{n}, X\right)$ if and only if $\alpha(\mathbf{1})=\alpha(f)+1$. Choose a subdivision of $\sigma$ such that the proper transform $\tilde{X}$ of $X$ under the corresponding toric morphism is nonsingular; $X$ could be resolved, for instance, by successive weighted blowups. By the adjunction formula, it follows that an exceptional divisor $E \subset \tilde{X}$ is crepant if and only if the corresponding weighting satisfies the equation $\alpha(\mathbf{1})=\alpha(f)+1$. This justifies the following:

Definition 3.1 A primitive weighting $\alpha \in \sigma \cap N$, not belonging to any proper face of $\sigma$ (see the remark below), is called crepant if it satisfies the equation

$$
\alpha(\mathbf{1})=\alpha(f)+1
$$

Denote by $W(f)$ the set of crepant weightings. 
Remark 3.2 The reason for restricting to interior weightings $\alpha \in \sigma^{\circ} \cap N$ in the above definition is that, since it is always possible to assume that $f$ contains a power of each variable, the only solution $\alpha$ of the equation (2) with, say, $\alpha_{i}=0$ is $\alpha=e_{j}^{*}$, for some $j$.

Remark 3.3 A weighting $\alpha \in \sigma \cap N$ satisfying (2) is automatically primitive.

A weighted blowup of a given singularity is not normal in general. Consider, for instance, the singularity $0 \in X:(f=0) \subset \mathbb{C}^{4}$, where

$$
f=x^{3}+y^{3}+z^{3}+t^{n} \quad \text { with } \quad n \geq 3,
$$

and $\alpha=(k, k, 1,1)$, for some $1 \leq k \leq[n / 3]$. One affine piece of the $\alpha$-blowup of $X$ is given by

$$
x=t^{k} x_{1}, \quad y=t^{k} y_{1} \quad \text { and } \quad z=t z_{1}
$$

and thus

$$
X(\alpha): t^{3(k-1)}\left(x_{1}^{3}+y_{1}^{3}\right)+z_{1}^{3}+t^{n-3}=0
$$

is not normal, unless $k=1$. Taking its normalisation by replacing $z_{1}$ with $z_{1} / t^{k-1}$ leads to the new weighting $\beta=(k, k, k, 1)$, which is crepant.

Nevertheless, if $\alpha$ is crepant, then $X(\alpha)$ is normal. This is the content of the following:

Proposition 3.4 Let $0 \in X \subset \mathbb{C}^{n}$ be a canonical hypersurface singularity and $\alpha$ a crepant weighting. Then the $\alpha$-blowup of $X$ also has canonical singularities.

Proof We only need to check that $X(\alpha)$ is normal. This is a direct consequence of the adjunction and subadjunction formulas (see [5]).

(1) Adjunction. From the relation (1) it follows, since $\alpha$ is crepant, that the sheaf $\omega_{\mathbb{C}^{n}(\alpha)}(X(\alpha))$ of rational sections of $\omega_{\mathbb{C}^{n}(\alpha)}$ with poles along $X(\alpha)$ satisfies

$$
\omega_{\mathbb{C}^{n}(\alpha)}(X(\alpha))=\varphi_{\alpha}^{*} \omega_{\mathbb{C}^{n}}(X)=\mathcal{O}_{\mathbb{C}^{n}(\alpha)} .
$$

Part of the long exact sequence of sheaves $\mathcal{E} x t^{i}\left(\cdot, \omega_{\mathbb{C}^{n}(\alpha)}\right)$ on $\mathbb{C}^{n}(\alpha)$ associated to the standard exact sequence

$$
0 \rightarrow \mathcal{I}_{X(\alpha)} \rightarrow \mathcal{O}_{\mathbb{C}^{n}(\alpha)} \rightarrow \mathcal{O}_{X(\alpha)} \rightarrow 0
$$


is the following:

$$
\mathcal{H o m}\left(\mathcal{I}_{X(\alpha)}, \omega_{\mathbb{C}^{n}(\alpha)}\right) \rightarrow \mathcal{E} x t^{1}\left(\mathcal{O}_{X(\alpha)}, \omega_{\mathbb{C}^{n}(\alpha)}\right) \rightarrow \mathcal{E} x t^{1}\left(\mathcal{O}_{\mathbb{C}^{n}(\alpha)}, \omega_{\mathbb{C}^{n}(\alpha)}\right)=0 .
$$

Since $\mathcal{H o m}\left(\mathcal{I}_{X(\alpha)}, \omega_{\mathbb{C}^{n}(\alpha)}\right)=\omega_{\mathbb{C}^{n}(\alpha)}(X(\alpha))$ and $\mathcal{E} x t^{1}\left(\mathcal{O}_{X(\alpha)}, \omega_{\mathbb{C}^{n}(\alpha)}\right)=$ $\omega_{X(\alpha)}$, it follows that

$$
\omega_{X(\alpha)}=\mathcal{O}_{X(\alpha)}
$$

(2) Subadjunction. Suppose $X(\alpha)$ is not normal and take its normalisation $\pi: \widetilde{X(\alpha)} \rightarrow X(\alpha)$. Let $C \subset \widetilde{X(\alpha)}$ be defined by $\mathcal{I}_{C}=\widetilde{\mathcal{C}}$, where

$$
\mathcal{C}=\operatorname{Ann}\left(\pi_{*} \mathcal{O}_{\widetilde{X(\alpha)}} / \mathcal{O}_{X(\alpha)}\right) \quad \text { and } \quad \widetilde{\mathcal{C}}=\mathcal{C} \mathcal{O}_{\widetilde{X(\alpha)}}
$$

as in $[5,2.1]$. Then $[5,2.3]$

$$
\pi^{*} \omega_{X(\alpha)}=\omega_{\widetilde{X(\alpha)}}(C) .
$$

Relations (3) and (4) with $C \neq 0$ contradict the assumption that $0 \in X$ is canonical.

In the remainder of this section we prove some lemmas which will be used in Section 4. Before giving the precise statements, we fix some notation and briefly describe how these lemmas will be used in the proof of Theorem 4.3.

Let $0 \in X:(f=0) \subset \mathbb{C}^{n}$ be a canonical singularity, and let $\alpha$ be a crepant weighting. Let $f=f_{\alpha}+f_{>\alpha}$ be the $\alpha$-homogeneous decomposition of $f$. Denote $f^{(j)}=\sum a_{m} x^{m}$, where the summation is taken over all monomials $m \in f$ with $m_{j}=0$; similar notation for $f_{\alpha}$ and $f_{>\alpha}$. Denote also by $E(\alpha)$ the exceptional locus of the $\alpha$-blowup of $X$.

The calculation of valuations along crepant divisors $E \subset E(\alpha)$ in the proof of Theorem 4.3 involves the choice of a monomial $m_{0} \in M$ with $\alpha\left(m_{0}\right)=1$. If $x_{j} \mid f_{\alpha}$ for some $j$ with $1 \leq j \leq n$, this calculation is simplified if such a monomial $m_{0}$ can be found with the additional property that its $j$ th component is zero. This can be done by Lemma 3.5. Denote

$$
l=\min _{m \in f_{\alpha}} m_{j} \quad \text { and } \quad l^{\prime}=\min _{m \in f_{>\alpha}^{(j)}}(\alpha(m)-\alpha(f)) .
$$

Lemma 3.6 will be used to express $v_{E}\left(x_{i}\right)$ in terms of $\alpha_{i}, l$ and $l^{\prime}$, for a divisor $E$ which is not essential for its valuation (see Definition 4.1). The values $v_{E}\left(x_{i}\right)$ define a new weighting $\beta$ by $\beta_{i}=v_{E}\left(x_{i}\right)$, which is crepant according to Lemma 3.7.

Lemma 3.5 Let $0 \in X:(f=0) \subset \mathbb{C}^{n}$ be a canonical singularity, and let $\alpha$ be a crepant weighting. Assume that $x_{j} \mid f_{\alpha}$ for some $j$ with $1 \leq j \leq n$. Then

$$
\operatorname{gcd}\left(\alpha_{1}, \ldots, \alpha_{j-1}, \alpha_{j+1}, \ldots, \alpha_{n}\right)=1 .
$$


Proof Let $d=\operatorname{gcd}\left(\alpha_{1}, \ldots, \alpha_{j-1}, \alpha_{j+1}, \ldots, \alpha_{n}\right)$ and denote $l=\min _{m \in f_{\alpha}} m_{j}$. By assumption, $l \geq 1$.

Define a new weighting $\beta \in \sigma^{\circ} \cap N$ by $\beta_{j}=\left\lceil\alpha_{j} / d\right\rceil$, and $\beta_{i}=\alpha_{i} / d$ for $i \neq j$, where, for $r$ a rational number, $\lceil r\rceil$ is the smallest integer $\geq r$.

Since $0 \in X$ is canonical, we have $\beta(\mathbf{1}) \geq \beta(f)+1$ (see [4, (4.8)]). This is equivalent to

$$
\alpha(\mathbf{1})+d \beta_{j}-\alpha_{j} \geq \min _{m \in f}\left(\alpha(m)+d \beta_{j} m_{j}-\alpha_{j} m_{j}\right)+d .
$$

Suppose there exists a monomial $m \in f_{>\alpha}$ such that

$$
\alpha(\mathbf{1})+d \beta_{j}-\alpha_{j} \geq \alpha(m)+\left(d \beta_{j}-\alpha_{j}\right) m_{j}+d .
$$

Since $\alpha$ is crepant, it follows that $d \beta_{j}-\alpha_{j} \geq\left(d \beta_{j}-\alpha_{j}\right) m_{j}+d$. Thus $m_{j}=0$, which gives $d \beta_{j}-\alpha_{j} \geq d$. This contradicts the definition of $\beta_{j}$.

It is enough, therefore, to consider in (5) only monomials $m \in f_{\alpha}$. Using again the fact that $\alpha$ is crepant, (5) becomes $d \beta_{j}-\alpha_{j}+1 \geq l\left(d \beta_{j}-\alpha_{j}\right)+d$. The last inequality is possible if and only if $d=1$.

Lemma 3.6 Assume that $x_{j} \mid f_{\alpha}$ for some $j$ with $1 \leq j \leq n$ and denote $l=\min _{m \in f_{\alpha}} m_{j}$ as above. Denote also

$$
l^{\prime}=\min _{m \in f_{>\alpha}^{(j)}}(\alpha(m)-\alpha(f)) .
$$

Then either $l$ or $l^{\prime}$ is equal to 1.

Proof Define $\beta \in \sigma^{\circ} \cap N$ by $\beta_{j}=\alpha_{j}+1$ and $\beta_{i}=\alpha_{i}$ for $i \neq j$. Since $\beta(\mathbf{1}) \geq \beta(f)+1$, at least one of the following conditions must hold:

(i) $\alpha(\mathbf{1}) \geq \min _{m \in f_{\alpha}}\left(\alpha(m)+m_{j}\right)$.

(ii) $\alpha(\mathbf{1}) \geq \min _{m \in f_{>\alpha}}\left(\alpha(m)+m_{j}\right)$.

Condition $(i)$ is equivalent to $\alpha(\mathbf{1}) \geq \alpha(f)+l$. In this case it follows that $l=1$.

Since $\min _{m \in f_{>\alpha}-f_{>\alpha}^{(j)}}\left(\alpha(m)+m_{j}\right) \geq \alpha(f)+2$ and $\alpha$ is crepant, (ii) becomes $\alpha(\mathbf{1}) \geq \min _{m \in f_{>\alpha}^{(j)}} \alpha(m)$. Using again the fact that $\alpha$ is crepant, it follows that $\alpha(f)+1 \geq \min _{m \in f_{>\alpha}^{(j)}} \alpha(m)$, and this is equivalent to $l^{\prime}=1$. 
Lemma 3.7 Same assumptions and notation as before. Then the new weighting $\beta \in \sigma \cap N$ defined by

$$
\beta_{i}=l \alpha_{i} \text { and } \beta_{j}=l \alpha_{j}+l^{\prime}
$$

is crepant.

Proof Using the fact that $\alpha$ is crepant it follows that

$$
\beta(\mathbf{1})=l \alpha(\mathbf{1})+l^{\prime}=l(\alpha(f)+1)+l^{\prime} .
$$

In order to express $\beta(f)$ in terms of $\alpha(f)$, notice that any monomial $m \in f_{>\alpha}$ with $m_{j} \neq 0$ satisfies $l \alpha(m)+l^{\prime} m_{j} \geq l(\alpha(f)+1)+l^{\prime}$. Furthermore

$$
\min _{m \in f_{\alpha}} \beta(m)=\min _{m \in f_{\alpha}}\left(l \alpha(m)+l^{\prime} m_{j}\right)=l \alpha(f)+l l^{\prime},
$$

and $\min _{m \in f_{>\alpha}^{(j)}} \beta(m)=l\left(\alpha(f)+l^{\prime}\right)$. Therefore $\beta(f)=l \alpha(f)+l l^{\prime}$, and the conclusion now follows from the previous lemma.

\section{Crepant valuations}

Recall that, given an isolated canonical nondegenerate singularity $0 \in X$ : $(f=0) \subset \mathbb{C}^{n}$, we are interested in the exceptional divisors $E(\alpha):=E_{\alpha} \cap$ $X(\alpha)$ on weighted blowups $X(\alpha)$ of $X$ with $\alpha$ crepant weightings, that is, weightings satisfying

$$
\alpha(\mathbf{1})=\alpha(f)+1 \text {. }
$$

In order to calculate the number $c(X)$ it is more convenient to use a birationally invariant language, namely that of geometric discrete valuations $v$ of the function field $k(X)$. To any prime divisor $E$ on a partial resolution of $X$, associate the corresponding valuation

$$
v_{E}: k(X)^{*} \rightarrow \mathbb{Z}
$$

By formula (1), for any crepant valuation $v$, there exists a crepant weighting $\alpha$ and a prime divisor $E \subset E(\alpha)$ such that $v=v_{E}$. This gives a relation between crepant valuations and exceptional prime divisors on weighted blowups with crepant weightings. In order to make this correspondence 1-to-1, we introduce the following: 
Definition 4.1 Let $\alpha$ be a crepant weighting. A prime divisor $E$ on the $\alpha$-blowup of $X$ is called essential for its valuation if $v_{E}\left(x_{i}\right)=\alpha_{i}$. Denote by $E(f)$ the set of divisors which are essential for their valuations.

In this section we prove that $E(f)$ is a set of representatives of the crepant valuations. Before stating the result, we give an illustration of the calculations involved in the proof of Theorem 4.3.

Example 4.2 The polynomial $f=x^{3}+x^{2} z+y^{2} z+z^{4}+t^{12}$ defines an isolated canonical nondegenerate singularity $0 \in X:(f=0) \subset \mathbb{C}^{4}$. There are 6 crepant weightings:

$$
W(f)=\{(1,1,1,1),(2,2,2,1),(3,3,2,1),(3,3,3,1),(4,4,3,1),(4,4,4,1)\} .
$$

Let $\alpha=(k, k, k, 1)$ with $1 \leq k \leq 4$. The $\alpha$-blowup of $X$ is given in one affine piece by setting $x=t^{k} x_{1}, y=t^{k} y_{1}$ and $z=t^{k} z_{1}$. Thus

$$
X(\alpha): x_{1}^{3}+x_{1}^{2} z_{1}+y_{1}^{2} z_{1}+t^{k} z_{1}^{4}+t^{12-3 k}=0 .
$$

In this case $t$ is a uniformising parameter and $x_{1}, y_{1}$ and $z_{1}$ are units of $\mathcal{O}_{X(\alpha), E(\alpha)}$. Therefore $E(\alpha) \in E(f)$.

If $\alpha=(3,3,2,1)$, the $\alpha$-blowup of $X$ is given by

$$
x=t^{3} x_{1} \quad y=t^{3} y_{1} \quad z=t^{2} z_{1} .
$$

The divisor $E(\alpha)$ has 2 irreducible components, $E_{1}:\left(x^{2}+y^{2}+z^{3}=0\right) \subset$ $\mathbb{P}(\alpha)$, which is essential for its valuation, and $E_{2}:(z=0) \subset \mathbb{P}(\alpha)$. The latter is given on

$$
X(\alpha): t x_{1}^{3}+x_{1}^{2} z_{1}+y_{1}^{2} z_{1}+z_{1}^{4}+t^{4}=0
$$

by $t=z_{1}=0$. Thus $v(x)=3, v(y)=3, v(z)=3$ and $v(t)=1$, where $v$ is the valuation along $E_{2}$. The weighting $\beta=(3,3,3,1)$ is crepant, and the divisors $E_{2}$ and $E(\beta)$ represent the same valuation.

Similarly, for $\alpha=(4,4,3,1)$, the divisor $E(\alpha):\left(x^{2} z+y^{2} z=0\right) \subset$ $\mathbb{P}(\alpha)$ has 3 irreducible components, and only 2 of them belong to $E(f)$. Calculating the valuation along $E:(z=0)$ gives $v(x)=4, v(y)=4$, $v(z)=4$ and $v(t)=1$. As in the previous case, the weighting $\beta=(4,4,4,1)$ is crepant, and $v=v_{E(\beta)}$.

Theorem 4.3 Let $0 \in X:(f=0) \subset \mathbb{C}^{n}$ be an isolated canonical nondegenerate singularity, $\alpha$ a crepant weighting, and $E$ an irreducible component of $E(\alpha) \subset \mathbb{P}(\alpha)$. Then 
(1) The divisor $E$ is essential for its valuation if and only if $E \subset \mathbb{P}(\alpha)$ is not one of the coordinate hyperplanes.

(2) Any crepant valuation is the valuation along a divisor in the set $E(f)$.

Proof The proof has two parts:

(A) The first part is based on explicit calculations of valuations along crepant divisors and proves (1); it also defines, for $E \notin E(f)$, a new weighting $\beta$, by $\beta_{i}=v_{E}\left(x_{i}\right)$.

(B) In the second part we prove that $E(\beta)$ has precisely one component $E^{\prime}$ which is essential for its valuation, and that $X(\alpha)$ and $X(\beta)$ are locally isomorphic over $X$ at the generic points of $E$ and $E^{\prime}$.

Part (A) Valuations along crepant divisors. Choose an arbitrary Laurent monomial $m_{0} \in M$ satisfying $\alpha\left(m_{0}\right)=1$ and let $u=x^{m_{0}}$ as in Section 2 . The $\alpha$-blowup of $\mathbb{C}^{n}$ is given on the open set $U_{\alpha}$ by $x_{i}=u^{\alpha_{i}} y_{i}$. Notice that the $y_{i}$ are related by $y^{m_{0}}=1$. Let $U:=X(\alpha) \cap U_{\alpha}$ be the proper transform of $X$ on $U_{\alpha}$. Its equation is

$$
\sum_{m \in f} u^{\alpha(m)-\alpha(f)} y^{m}=0
$$

On $U$ the exceptional divisor $E(\alpha)$ is given by $u=f_{\alpha}(y)=0$. Let $g$ be the irreducible polynomial such that the component $E$ has the expression

$$
E: u=g(y)=0 \text { on the open set } U \text {. }
$$

Denote by $A=\mathcal{O}_{X(\alpha), E}$ the localisation of the ring of regular function on $X(\alpha)$ at the generic point of $E$. Denote also by $v=v_{E}$ the corresponding valuation.

If $g(y) \neq y_{i}$ for any $i$, it follows from the nondegeneracy assumption that $u$ is a uniformising parameter and all the $y_{i}$ are units in $A$. Therefore $v\left(x_{i}\right)=\alpha_{i}$, i.e., $E$ is essential.

Suppose that $g(y)=y_{j}$ for some $j$. Then $v\left(y_{j}\right) \geq 1$ and $E$ is no longer essential. This proves (1).

Let now $l=\min _{m \in f_{\alpha}} m_{j}$ and choose a monomial $m_{0}$ as above with the additional property that its $j$ th component is zero. Such a monomial exists by Lemma 3.5 . In the local ring $A$, relation (6) becomes

$$
\sum_{m \in f_{>\alpha}} u^{\alpha(m)-\alpha(f)} y^{m}=-\sum_{m \in f_{\alpha}} y^{m}=y_{j}^{l} \text { (unit). }
$$

We have to distinguish two cases: 
(a) $l \geq 2$. In this case it follows from Lemma 3.6 that

$$
l^{\prime}:=\min _{m \in f_{>\alpha}^{(j)}}(\alpha(m)-\alpha(f))=1
$$

and (7) reads $u=y_{j}^{l}$ (unit). This implies that $v\left(y_{j}\right)=1$ and $v(u)=l$; thus

$$
v\left(x_{j}\right)=l \alpha_{j}+1 \text { and } v\left(x_{i}\right)=l \alpha_{i} \text { if } i \neq j .
$$

The new weighting $\beta$ defined by

$$
\beta_{j}=l \alpha_{j}+1 \text { and } \beta_{i}=l \alpha_{i} \text { if } i \neq j
$$

is crepant by Lemma 3.7 .

(b) $l=1$. In this case (7) becomes

$$
\sum_{m \in f_{>\alpha}^{(j)}} u^{\alpha(m)-\alpha(f)} y^{m}+\sum_{m \in f>\alpha-f_{>\alpha}^{(j)}} u^{\alpha(m)-\alpha(f)} y^{m}=y_{j} \text { (unit) }
$$

and this reduces to $u^{l^{\prime}}=y_{j}$ (unit). Thus $u$ is a local parameter, $v(u)=1$, $v\left(y_{j}\right)=l^{\prime}$ and

$$
v\left(x_{j}\right)=\alpha_{j}+l^{\prime}, \quad v\left(x_{i}\right)=\alpha_{i}, \text { if } i \neq j .
$$

As in the previous case, define a new weighting $\beta$ by

$$
\beta_{j}=\alpha_{j}+l^{\prime}, \quad \beta_{i}=\alpha_{i}, \text { if } i \neq j .
$$

Again $\beta$ is crepant by Lemma 3.7.

Part (B) The local isomorphism. Let the monomial $m_{0}^{\prime} \in M$ with $\beta\left(m_{0}^{\prime}\right)=1$ be defined by

$$
\begin{aligned}
& m_{0}^{\prime}=e_{j}^{*}-\alpha_{j} m_{0}, \quad \text { in case }(a), \\
& m_{0}^{\prime}=m_{0}, \quad \text { in case }(b) .
\end{aligned}
$$

Define $u^{\prime}=x^{m_{0}^{\prime}}$ and $z_{i}=\left(u^{\prime}\right)^{-\beta_{i}} x_{i}$, and let $U_{\beta}^{\prime}=\operatorname{Spec} \mathbb{C}\left[u^{\prime}, z_{1}, \ldots, z_{n}\right]$ and $U^{\prime}:=X(\beta) \cap U_{\beta}^{\prime}$. Look at the birational map $\varphi_{\beta \alpha}$ over $X$ between $U^{\prime}$ and $U$

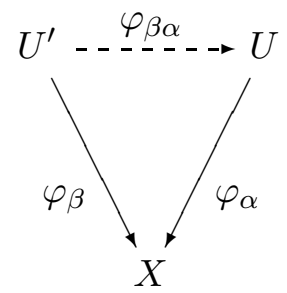


This map, restricted to the locus $U^{\prime} \backslash\left\{\prod_{i=1}^{n} z_{i}=0\right\}$, turns out to be a morphism, as the following calculation shows. We have $x_{i}=u^{\alpha_{i}} y_{i}=\left(u^{\prime}\right)^{\beta_{i}} z_{i}$ and, taking the product over all $i$, it follows that $u^{\alpha\left(m_{0}\right)}=\left(u^{\prime}\right)^{\beta\left(m_{0}\right)} z^{m_{0}} y^{-m_{0}}$. Since $\alpha\left(m_{0}\right)=1, y^{m_{0}}=1$ and $\beta\left(m_{0}\right)=l$, this implies that the map $\varphi_{\beta \alpha}$ is given by

$$
\begin{aligned}
& y_{j}=u^{\prime}, u=\left(u^{\prime}\right)^{l} z^{m_{0}}, y_{i}=z_{i} z^{-\alpha_{i} m_{0}}, \quad \text { in case }(a), \\
& y_{j}=\left(u^{\prime}\right)^{l^{\prime}} z_{j}, u=u^{\prime}, y_{i}=z_{i}, \quad \text { in case }(b),
\end{aligned}
$$

and therefore is a morphism.

Take a monomial $m \in f$; we have

$$
m \in f_{\beta} \Longleftrightarrow \begin{cases}\text { either } & m \in f_{\alpha} \text { and } m_{j}=l, \\ \text { or } & m \in f_{>\alpha}^{(j)} \text { and } \alpha(m)=\alpha(f)+l^{\prime} .\end{cases}
$$

This shows, in particular, that $\operatorname{dim} \Gamma_{\beta} \geq 1$; let $E^{\prime}$ be any essential component of $E(\beta)$. The morphism $\varphi_{\beta \alpha}$ gives an injective morphism

$$
\varphi_{\beta \alpha}^{*}: A \rightarrow B,
$$

where $B:=\mathcal{O}_{X(\beta), E^{\prime}}$ is the localisation of the ring of regular function on $X(\beta)$ at the generic point of $E^{\prime}$. Therefore $B$ dominates $A$ for any choice of $E^{\prime}$, which gives the desired isomorphism. This completes the proof.

Remark 4.4 Let $v$ be a crepant valuation and denote $\beta_{i}=v\left(x_{i}\right)$. There are finitely many weightings $\alpha \in W(f)$ such that $v=v_{E}$ for some irreducible component $E \subset E(\alpha)$. This follows immediately from part (A) of the above proof. Indeed, if $E \subset \mathbb{P}(\alpha)$ is not one of the coordinate hyperplanes, then $E \in E(f)$ and $\alpha=\beta$. Otherwise $l \alpha_{j}+l^{\prime}=\beta_{j}$ for some $j$, and $l \alpha_{i}=\beta_{i}$ for all $i \neq j$.

Since the set of crepant valuations is finite, it follows that $W(f)$ is also finite.

\section{A formula for $c(X)$}

Let $0 \in X:(f=0) \subset \mathbb{C}^{n}$ be an isolated canonical nondegenerate singularity, $\alpha \in \sigma^{\circ} \cap N$ a primitive weighting, and

$$
f_{\alpha}=\prod_{i=1}^{n} x_{i}^{l_{i}} \prod_{j=1}^{c(\alpha)} g_{j}
$$


the decomposition of $f_{\alpha}$ into irreducible components. From Theorem 4.3 it follows that

$$
c(X)=\sum_{\alpha \in W(f)} c(\alpha)
$$

This formula becomes more precise once we notice that

$$
c(\alpha)= \begin{cases}\operatorname{length} \Gamma_{\alpha} & \text { if } \operatorname{dim} \Gamma_{\alpha}=1 \\ 1 & \text { if } \operatorname{dim} \Gamma_{\alpha} \geq 2\end{cases}
$$

where $\Gamma_{\alpha}$ denotes the face of $\Gamma(f)$ corresponding to $\alpha$, and length $\Gamma_{\alpha}=$ $\#\left(M \cap \Gamma_{\alpha}\right)-1$. For the case $\operatorname{dim} \Gamma_{\alpha} \geq 2$ it is enough, for instance, to view $\left(f_{\alpha}=0\right)$ as a hypersurface in the torus $\left(\mathbb{C}^{*}\right)^{n}$, and then apply the toric Lefschetz property proved in [2]. Thus we have proved the following:

Corollary 5.1 Let $0 \in X:(f=0) \subset \mathbb{C}^{n}$ be an isolated canonical nondegenerate singularity. Then the number of crepant divisors $c(X)$ is given by

$$
c(X)=\sum_{\substack{\alpha \in W(f) \\ \operatorname{dim} \Gamma_{\alpha}=1}} \operatorname{length} \Gamma_{\alpha}+\#\left\{\alpha \in W(f): \operatorname{dim} \Gamma_{\alpha} \geq 2\right\} .
$$

Example 5.2 Let $0 \in X:(f=0) \subset \mathbb{C}^{4}$ where $f=x^{3}+x^{2} z+y^{2} z+z^{4}+t^{12}$ as in Example 4.2. The only crepant weighting $\alpha$ with $\operatorname{dim} \Gamma_{\alpha}=1$ is $\alpha=$ $(4,4,3,1)$. In this case, length $\Gamma_{\alpha}=2$. By Corollary 5.1 , the number of crepant divisors of $X$ is $c(X)=7$.

\section{References}

[1] M. Caibăr, 'Minimal models of canonical singularities and their cohomology', Ph.D. Thesis, University of Warwick, 1999, 78 + vii pp.

[2] V. I. Danilov and A. G. Khovanskiı,, 'Newton polyhedra and an algorithm for calculating Hodge-Deligne numbers', Math. USSR Izv. 29 (1987), 279-298

[3] M. Reid, 'Canonical 3-folds', in Journées de géometrie algébrique d'Angers, 273-310, Sijthoff and Noordhoff, Alphen aan den Rijn, 1980 
[4] M. Reid, 'Young person's guide to canonical singularities', in Algebraic geometry, Bowdoin, 1985 , 345-414, Proc. Sympos. Pure Math., Part 1,1987

[5] M. Reid, 'Nonnormal del Pezzo surfaces', Publ. Res. Inst. Math. Sci. 30 (1994), no. 5, 695-727

Department of Mathematics

University of North Carolina at Chapel Hill

Chapel Hill, NC 27599, U.S.A.

E-mail: mcaibar@math.unc.edu 